\title{
A genetic link between type 2 diabetes and prostate cancer
}

\author{
T. M. Frayling $・$ H. Colhoun • J. C. Florez
}

Received: 4 July 2008 / Accepted: 7 July 2008 / Published online: 12 August 2008

(C) Springer-Verlag 2008

\begin{abstract}
Epidemiological studies suggest that men with type 2 diabetes are less likely than non-diabetic men to develop prostate cancer. The cause of this association is not known. Recent genetic studies have highlighted a potential genetic link between the two diseases. Two studies have identified a version (allele) of a variant in the HNF1B (also known as $T C F 2$ ) gene that predisposes to type 2 diabetes, and one of them showed that the same allele protects men from prostate cancer. Other, separate, studies have identified different variants in the $J A Z F 1$ gene, one associated with type 2 diabetes, another associated with prostate cancer. These findings are unlikely to completely explain the epidemiological association between the two diseases but they provide new insight into a possible direct causal link, rather than one that is confounded or biased in some way.
\end{abstract}

Keywords Epidemiology · Genes ·

Genome-wide association study · Pleiotropy ·

Prostate cancer · Transcription factors · Type 2 diabetes

T. M. Frayling $(\bowtie)$

Genetics of Complex Traits,

Peninsula College of Medicine and Dentistry,

University of Exeter,

Magdalen Road,

Exeter EX1 2LU, UK

e-mail: Tim.frayling@pms.ac.uk

H. Colhoun

University of Dundee,

Dundee, UK

J. C. Florez

Diabetes Unit and Center for Human Genetic Research,

Massachusetts General Hospital,

Boston, MA, USA

\begin{abstract}
Abbreviations
PSA prostate-specific antigen

SNP single nucleotide polymorphism
\end{abstract}

\section{Prostate cancer, BMI and type 2 diabetes}

There is extensive but conflicting literature on whether diabetes mellitus is associated with protection from prostate cancer. The extent to which bias and confounding factors could influence this association is also unclear. The most comprehensive meta-analysis performed to date found an inverse association between diabetes and prostate cancer (RR 0.84, 95\% CI 0.76-0.93) [1], but there was significant heterogeneity between studies $(p<0.01)$. Adjustment for covariates, including BMI, strengthened the association. Two studies reported since this meta-analysis both showed evidence for a reduced risk of prostate cancer in men with type 2 diabetes. The Prostate Cancer Prevention Trial found that diabetes was associated with a $47 \%$ reduced risk of low-grade prostate cancer and a $28 \%$ reduced risk of high-grade prostate cancer [2]. In another large prospective cohort analysis, diabetes was associated with a $31 \%$ lower risk of prostate cancer [3].

Some data suggest that a reduced incidence of prostate cancer is more pronounced in those with a longstanding elevated risk reported in the first few years after the diagnosis of diabetes $[4,5]$. However, another study found the opposite; in the 1959-1972 Cancer Prevention Study there was no overall association between diabetes and prostate cancer incidence, but those with diabetes for 5 or more years had a higher incidence of prostate cancer than did men without diabetes [6].

The data on the association of either obesity or metabolic syndrome with prostate cancer incidence are also inconsistent. Both elevated and reduced risks have been reported in 
large, well-designed studies [7-10]. A recent meta-analysis concluded that the relative risk was 1.05 for every $5 \mathrm{~kg} / \mathrm{m}^{2}$ increment in BMI [11]. There is a suggestion that increased BMI is more strongly associated with more severe prostate cancer [11]. For example, in the Prostate Cancer Prevention trial, a BMI of $>30 \mathrm{~kg} / \mathrm{m}^{2}$ was associated with an $18 \%$ lower risk of low-grade prostate cancer and a $29 \%$ higher risk of high-grade prostate cancer. However, although all those reaching the end of the trial were offered a prostate biopsy, the protocol referred those with elevations in prostatespecific antigen (PSA) for biopsy earlier in the trial. These data are therefore also consistent with higher BMI being associated with a lower PSA level, which delays diagnosis until disease is more advanced [2]. An increased risk specific to more aggressive disease was also reported in the recent meta-analysis by MacInnis and English [11].

Diabetes might be associated with prostate cancer in a number of ways; risk factors for one may confer an increased or decreased risk of the other. Mechanisms proposed include differences in insulin levels, bioavailable IGF-1, leptin, free testosterone and drug treatments [8]. Relevant here is that there is some evidence to suggest that oral hypoglycaemic drugs such as metformin may reduce cancer risk [12, 13], and such a hypothesis would be consistent with the greater reduction in risk with longer duration of diabetes observed in some studies $[4,5]$.

It is also possible that the epidemiological association between type 2 diabetes and prostate cancer could reflect various types of unmeasured biases and confounding factors. For example, if cardiovascular or renal disease and prostate cancer share common risks, then depletion of diabetic cohorts by early mortality will lead to an apparent lower rate of prostate cancer in diabetes survivors. Other, more likely, biases include diagnostic bias, which may be introduced by the less than $100 \%$ sensitivity and specificity of different tests, such as PSA screening. In the National Health and Nutrition Examination Survey (NHANES) II, those with diabetes had a $21.6 \%$ lower mean PSA level than men without diabetes, and the difference increased with years since diagnosis [14]. This could reflect a true inverse association with prostate cancer; alternatively, it might cause a downward bias in the risk ratio for prostate cancer in type 2 diabetic men because fewer diabetic patients would proceed to diagnostic biopsy. Whether risk or severity of prostate cancer between diabetic and non-diabetic men differs for a given level of PSA is uncertain.

\section{Genome-wide association studies may provide insight into the overlap between different diseases}

Genome-wide association studies have recently identified many new common gene variants associated with prevalent diseases, including type 2 diabetes $[15,16]$. Most of the genes implicated were not obvious candidates and so provide new insight into similarities in disease aetiology. An additional, important message has emerged from the genome-wide association studies concerning the genetic overlap between different conditions. This is seen most obviously with respect to the autoimmune diseases. For example, several common (non-HLA) variants predispose to type 1 diabetes and at least one other autoimmune disease, including Crohn's disease, multiple sclerosis and coeliac disease [17-20].

\section{Two gene loci, three variants and two diseases}

Findings from several type 2 diabetes and prostate cancer genetic studies now show a convincing genetic link between the two diseases. First, Winckler and colleagues identified a common single nucleotide polymorphism (SNP) rs757210 in the HNF1B (also known as TCF2) gene that was robustly $\left(p=5 \times 10^{-6}\right)$ yet modestly (OR $1.12,95 \%$ CI 1.07 1.18) associated with type 2 diabetes [21]. Shortly thereafter, Gudmundsson et al. identified a common variant in the same gene associated with prostate cancer [22]. The A allele at this variant, situated in intron 2 of $H N F 1 B$ (Fig. 1a), was associated with an increased risk of prostate cancer with an OR of $1.22(95 \%$ CI $1.15-1.30 ; p=1.4 \times$ $\left.10^{-11}\right)$. This finding, together with the knowledge that rare mutations in HNF1B lead to MODY [23], led the researchers to investigate the variant in type 2 diabetes. The same allele of the same variant was associated with protection from type 2 diabetes (OR $0.91,95 \%$ CI $0.87-0.94, p=$ $\left.2.7 \times 10^{-7}\right)$. The SNPs investigated in the studies conducted by Winckler et al. and Gudmundsson et al., rs757210 and rs4430796, are highly correlated with each other (in linkage disequilibrium). Combining information from these two diabetes studies indicates that the risk allele for prostate cancer protects from type 2 diabetes with an OR of 0.91 (95\% CI $\left.0.88-0.93, p=8 \times 10^{-10}\right)$ [16]. Both the type 2 diabetes and the prostate cancer associations far exceed the stringent levels of statistical significance required in genome-wide studies $\left(p \approx 5 \times 10^{-8}\right)$.

A second locus is implicated in both prostate cancer and type 2 diabetes. Thomas et al. [24] recently reported the results of a genome-wide search for prostate cancer gene variants, which was similar in design to the Gudmundsson et al. study [22] and replicated the HNF1B findings. The authors also found that carriers of two copies of the $G$ allele at SNP rs10486567, situated in intron 2 of the JAZF1 gene (Fig. 1b), protected individuals from prostate cancer with ORs of 0.71 (95\% CI $0.55-0.90)$ and 0.84 (95\% CI $0.67-$ 1.05 ) for non-aggressive and aggressive forms of the disease, respectively (combined $p=2.4 \times 10^{-6}$ ) [24]. At the same time, an independent genome-wide study of type 2 diabetes 
a

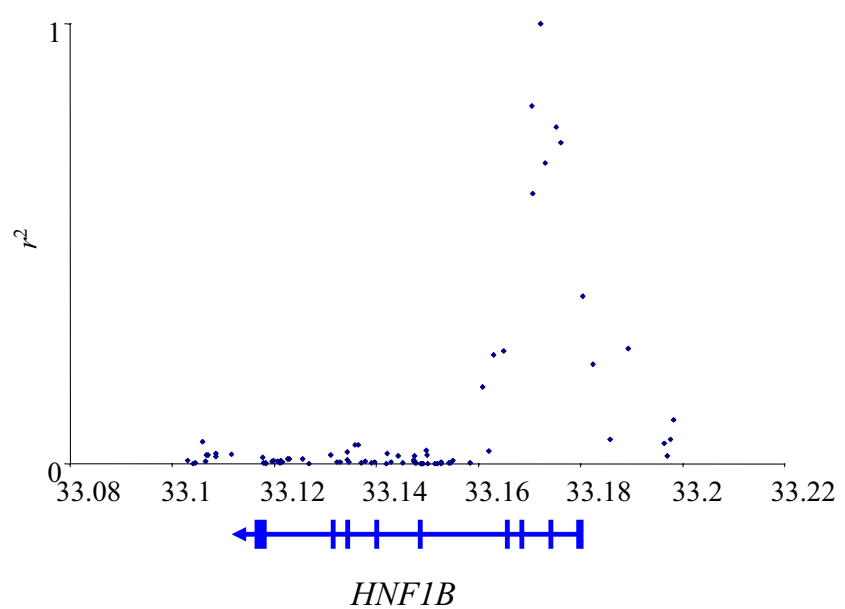

b

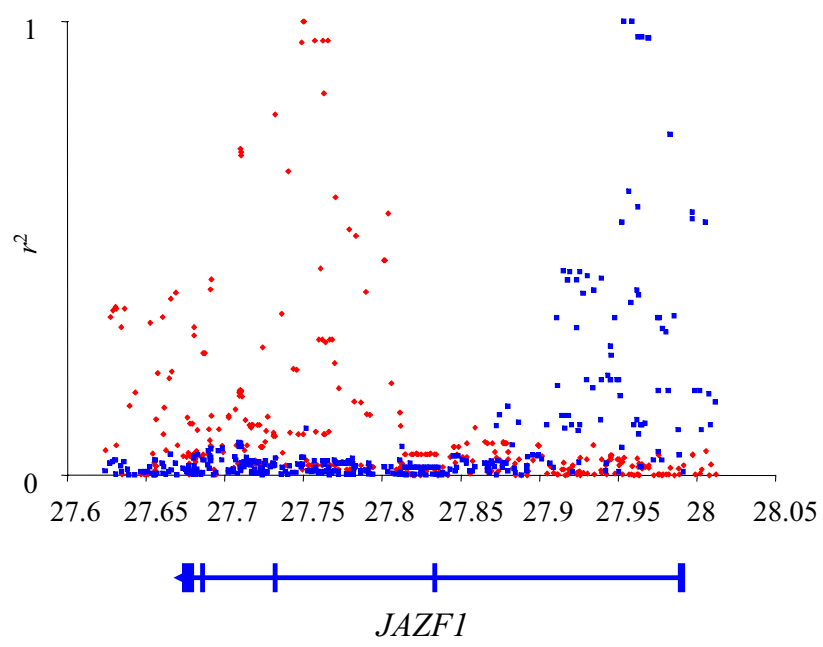

Fig. 1 Position of SNPs associated with prostate cancer and type 2 diabetes in the $H N F 1 B$ gene $\pm 20 \mathrm{~kb}$ (a), and $J A Z F 1$ gene $\pm 20 \mathrm{~kb}$ (b), together with positions of all other common ( $>5 \%$ in Europeans) SNPs in the European HapMap sample ( $x$-axis) and the degree of correlation with the most strongly associated SNPs, expressed as $r^{2}$ ( $y$-axis). For the $J A Z F 1$ gene, blue squares represent the type 2 diabetes signal and red diamonds the prostate cancer signal

identified a variant in the $J A Z F 1$ gene that predisposed to type 2 diabetes with an OR of $1.10(95 \%$ CI $1.08-1.14, p=$ $8.8 \times 10^{-12}$ ) [15]. The two SNPs reported were not the same, but in the aftermath of the $H N F 1 B$ finding, the obvious question was whether or not the two variants were correlated with each other and so represented the same genetic signal (as a result of linkage disequilibrium). The answer was negative - the type 2 diabetes and prostate cancer variants are not correlated. This implies that the variants (or those correlated with them) may be operating in completely different ways, and perhaps even on different genes. Nevertheless, the findings from the two loci provide strong evidence that variation in the same genes could affect the risk of these two diseases.

\section{How could DNA variation in the $H N F 1 B$ and $J A Z F 1$ genes influence two diseases?}

The recent discovery of these variants means that we know little about how the DNA variation in the $H N F 1 B$ and $J A Z F 1$ genes influence two diseases. We cannot even be certain that the variants alter these genes-it is possible that SNPs in one gene are involved in the control of a second, neighbouring gene. We can therefore only speculate as to the likely mechanisms involved. What we do know is that both gene products are transcription factors that control the expression of many other genes. Both of them are involved in early development. Thus, these genetic associations provide further evidence for the role of general, often early, gene transcription in the aetiology of diabetes. To what extent these genes participate in the long-term maintenance of beta cell mass is unknown. It is also not unusual for transcription factors, or other genes involved in transcriptional processes, to be involved in cancer progression, presumably as a result of the abnormal expression of genes downstream in the pathway.

Studies of $H N F 1 B$ allow us to speculate about how this transcription factor may be involved in both diseases. We know most about the role of $H N F 1 B$ in diabetes because of the work following its discovery as a MODY gene, and later as a gene involved in renal disease and other abnormalities [25]. HNF1B is also involved in neoplasia. For example, a combination of germline and somatic $H N F 1 B$ mutations have been reported in two cases of chromophobe renal cell carcinoma [26]. This means that $H N F 1 B$ can be classified as a tumour suppressor gene in the kidney. The recent genetic associations with prostate cancer suggest that it can also play this role in the prostate.

There are links between JAZF1 and cancer, but little is known about its possible role in type 2 diabetes. Translocations that result in a fusion protein involving $J A Z F 1$ and SUZ12 (also known as $J J A Z 1$ ) are found in endometrial stromal cell tumours [27]. SUZ12 encodes a 'polycomb' protein - a class of proteins involved in chromatin remodelling (the unravelling of the tightly coiled DNA) to control gene expression. The best clue as to the role of JAZF1 in type 2 diabetes comes from the fact that it is involved in the expression of $N R 2 C 2$, which encodes nuclear receptor subfamily 2 , group $\mathrm{C}$, member 2 . Mice lacking $N R 2 C 2$ have neonatal and postnatal hypoglycaemia [28], a phenotype also observed in humans with $H N F 4 A$ mutations before they develop diabetes in later life [29].

More generally, the genetic connection between type 2 diabetes and cancer illustrates a possible scenario in which genes involved in cell cycle proliferation, when suppressed, may lead to inadequate beta cell mass in the adult, giving rise to insufficient insulin secretion and consequent diabetes; conversely, when overactivated in other tissues they 
might predispose to uncontrolled cellular proliferation. As the genes for more transcription factors (e.g. TCF7L2, $H H E X$ ) and cell cycle proteins (e.g. $C D K A L 1, C D K N 2 A / B$ ) become associated with diabetes, it will be interesting to explore their possible multiple effects in the various genome-wide cancer datasets.

\section{Conclusion}

The cause of the epidemiological association between type 2 diabetes and prostate cancer is not known. However, it is now clear that some genes may be involved in both diseases, and, in the case of $H N F 1 B$, a single common polymorphism provides a direct link. Consistent with the epidemiological data, the risk allele for type 2 diabetes is protective for prostate cancer, and vice versa. Further work is needed to assess possible additional genetic links between the two diseases. This intriguing observation may illustrate a general biological phenomenon connecting endocrine pancreatic insufficiency with the inherited susceptibility to neoplasia.

Duality of interest The authors are not aware of any duality of interest associated with this manuscript.

\section{References}

1. Kasper JS, Giovannucci E (2006) A meta-analysis of diabetes mellitus and the risk of prostate cancer. Cancer Epidemiol Biomarkers Prev 15:2056-2062

2. Gong Z, Neuhouser ML, Goodman PJ et al (2006) Obesity, diabetes, and risk of prostate cancer: results from the prostate cancer prevention trial. Cancer Epidemiol Biomarkers Prev 15: 1977-1983

3. Calton BA, Chang SC, Wright ME et al (2007) History of diabetes mellitus and subsequent prostate cancer risk in the NIH-AARP Diet and Health Study. Cancer Causes Control 18:493-503

4. Giovannucci E, Rimm EB, Stampfer MJ, Colditz GA, Willett WC (1998) Diabetes mellitus and risk of prostate cancer (United States). Cancer Causes Control 9:3-9

5. Rodriguez C, Patel AV, Mondul AM, Jacobs EJ, Thun MJ, Calle EE (2005) Diabetes and risk of prostate cancer in a prospective cohort of US men. Am J Epidemiol 161:147-152

6. Will JC, Vinicor F, Calle EE (1999) Is diabetes mellitus associated with prostate cancer incidence and survival. Epidemiology 10: 313-318

7. Hsing AW, Sakoda LC, Chua S Jr (2007) Obesity, metabolic syndrome, and prostate cancer. Am J Clin Nutr 86:S843-S857

8. Giovannucci E, Michaud D (2007) The role of obesity and related metabolic disturbances in cancers of the colon, prostate, and pancreas. Gastroenterology 132:2208-2225

9. Tande AJ, Platz EA, Folsom AR (2006) The metabolic syndrome is associated with reduced risk of prostate cancer. Am J Epidemiol 164:1094-1102
10. Lund Håheim L, Wisloff TF, Holme I, Nafstad P (2006) Metabolic syndrome predicts prostate cancer in a cohort of middle-aged Norwegian men followed for 27 years. Am J Epidemiol 164:769774

11. MacInnis RJ, English DR (2006) Body size and composition and prostate cancer risk: systematic review and meta-regression analysis. Cancer Causes Control 17:989-1003

12. Evans JM, Donnelly LA, Emslie-Smith AM, Alessi DR, Morris $\mathrm{AD}$ (2005) Metformin and reduced risk of cancer in diabetic patients. BMJ 330:1304-1305

13. Huang X, Wullschleger S, Shpiro N et al (2008) Important role of the LKB1-AMPK pathway in suppressing tumorigenesis in PTEN-deficient mice. Biochem J 412:211-221

14. Werny DM, Saraiya M, Gregg EW (2006) Prostate-specific antigen values in diabetic and nondiabetic US men, 2001-2002. Am J Epidemiol 164:978-983

15. Zeggini E, Scott LJ, Saxena R et al (2008) Meta-analysis of genome-wide association data and large-scale replication identifies additional susceptibility loci for type 2 diabetes. Nat Genet 40:638-645

16. Frayling TM (2007) Genome-wide association studies provide new insights into type 2 diabetes aetiology. Nat Rev Genet 8:657662

17. Todd JA, Walker NM, Cooper JD et al (2007) Robust associations of four new chromosome regions from genome-wide analyses of type 1 diabetes. Nat Genet 39:857-864

18. Parkes M, Barrett JC, Prescott NJ et al (2007) Sequence variants in the autophagy gene $I R G M$ and multiple other replicating loci contribute to Crohn's disease susceptibility. Nat Genet 39:830-832

19. Burton PR, Clayton DG, Cardon LR et al (2007) Association scan of 14,500 nonsynonymous SNPs in four diseases identifies autoimmunity variants. Nat Genet 39:1329-1337

20. Gregory SG, Schmidt S, Seth P et al (2007) Interleukin 7 receptor a chain $(I L 7 R)$ shows allelic and functional association with multiple sclerosis. Nat Genet 39:1083-1091

21. Winckler W, Weedon MN, Graham RR et al (2007) Evaluation of common variants in the six known maturity-onset diabetes of the young (MODY) genes for association with type 2 diabetes. Diabetes 56:685-693

22. Gudmundsson J, Sulem P, Steinthorsdottir V et al (2007) Two variants on chromosome 17 confer prostate cancer risk, and the one in TCF2 protects against type 2 diabetes. Nat Genet 39:977-983

23. Horikawa Y, Iwasaki N, Hara M et al (1997) Mutation in hepatocyte nuclear factor-1b gene (TCF2) associated with MODY. Nature Genetics 17:384-385

24. Thomas G, Jacobs KB, Yeager M et al (2008) Multiple loci identified in a genome-wide association study of prostate cancer. Nat Genet 40:310-315

25. Edghill EL, Bingham C, Ellard S, Hattersley AT (2006) Mutations in hepatocyte nuclear factor-1beta and their related phenotypes. J Med Genet 43:84-90

26. Rebouissou S, Vasiliu V, Thomas C et al (2005) Germline hepatocyte nuclear factor 1alpha and 1beta mutations in renal cell carcinomas. Hum Mol Genet 14:603-614

27. Li H, Ma X, Wang J, Koontz J, Nucci M, Sklar J (2007) Effects of rearrangement and allelic exclusion of JJAZ1/SUZ12 on cell proliferation and survival. Proc Natl Acad Sci USA 104:20001-20006

28. Nakajima T, Fujino S, Nakanishi G, Kim YS, Jetten AM (2004) TIP27: a novel repressor of the nuclear orphan receptor TAK1/ TR4. Nucleic Acids Res 32:4194-4204

29. Pearson ER, Boj SF, Steele AM et al (2007) Macrosomia and hyperinsulinaemic hypoglycaemia in patients with heterozygous mutations in the HNF4A gene. PLoS Med 4:e118 